0031 LUNG FUNCTION IMPROVEMENT IS SUSTAINED AFTER WORK CESSATION IN SHANGHAI COTTON AND SILK TEXTILE WORKERS

${ }^{1}$ Peggy Lai, ${ }^{2}$ Jing-qing Hang, ${ }^{2}$ Feng-ying Zhang, ${ }^{2}$ Bu-yong Zheng, ${ }^{1}$ Linda Valeri, ${ }^{3}$ Ellen Eisen, 'David Christiani. ${ }^{1}$ Harvard School of Public Health, Boston, MA, USA; ${ }^{2}$ Shanghai Putuo District People's Hospital, Shanghai, China; ${ }^{3}$ School of Public Health, University of California, Berkeley, CA, USA

\subsection{6/oemed-2014-102362.7}

Objectives Whether cessation of exposure to endotoxin containing organic dusts leads to transient vs. sustained improvement of lung function is unknown.

Method The Shanghai Textile Workers study is a 30 -year prospective cohort study of 447 cotton workers exposed to endotoxin containing cotton dust and 472 control silk workers unexposed to endotoxin. Spirometry and questionnaires were administered at 5 year intervals, and endotoxin sampling was performed to estimate individual cumulative exposures. The effect of work cessation on FEV1 was modelled with a generalised additive mixed effects (GAMM) model.

Results When cessation was modelled as a smoothed term, adjusting for age, gender, height, and smoking history, cessation was associated with a significant FEV1 improvement in both cotton and silk workers. Work cessation displayed a non-linear quadratic effect on FEV1, with an average adjusted +38.1, $+220,+316 \mathrm{ml}$ effect in silk and $+26.3,+184.1,+264.1 \mathrm{ml}$ effect in cotton workers at 10, 20, and 25 years of work cessation. In a model allowing for a quadratic effect of cessation years as suggested by the GAMM model, the linear component of the interaction suggested that cessation of cotton work was associated with less FEV1 improvement than silk work (cotton*cessation year interaction $\beta=-2.6 \mathrm{ml}, \mathrm{p}=0.025$ ).

Conclusions Lung function improvement after work cessation was observed in both cotton and silk workers, suggesting that non-endotoxin containing organic dust exposure has adverse respiratory effects. The greater improvement in FEV1 in silk compared to cotton textile workers suggests that the endotoxin component of cotton dust is associated with additional detrimental effects.

\section{ASSOCIATIONS OF STRESS, ANXIETY, AND RESILIENCY IN POLICE WORK}

${ }^{1}$ John Violanti, ${ }^{2}$ Anna Mnatskanova, ${ }^{2}$ Andrew Michael, ${ }^{2}$ Hartley Tara, ${ }^{2}$ Fekedulegn Desta, ${ }^{2}$ Baughman Penelope, ${ }^{2}$ Burchfiel Cecil. 'University at Buffalo, State University of New York, Buffalo, NY, USA; ${ }^{2}$ Biostatistics and Epidemiology Branch, Health Effects Laboratory Division, National Institute for Occupational Safety and Health, Morgantown, WV, USA

\subsection{6/oemed-2014-102362.8}

Objectives Police work is an occupation replete with stress. The present study examined associations between specific police stressors (overall, administrative pressure, physical threat, and lack of support) and anxiety symptoms, and whether these associations were modified by hardiness, a dimension of resiliency.

Method The Spielberger Police Stress Survey, Beck Anxiety Scale, and Dispositional Resilience scale were utilised in this study. A total of 373 police officers with complete data from the Buffalo Cardio-Metabolic Occupational Police Stress (BCOPS) study were included. Linear regression and analysis of covariance were used to examine mean anxiety levels across quartiles of stress. Associations were adjusted for age, sex, race, alcohol, smoking, and anxiety medication, and stratified by hardiness scores.

Results The mean age of officers was 41.4 years and 27\% were female. Adjusted mean anxiety symptoms increased significantly with increasing stress quartiles overall $(4.23,4.99,6.74$ and 9.95 for quartiles $1-4$, respectively, $\mathrm{p}<0.001$ ) and for all three types of stressors $(p<0.001)$. Hardiness did not significantly modify these associations. However, officers with hardiness scores above the median had generally lower anxiety scores than those below the median.

Conclusions Specific types of stress in police work are significantly associated with symptoms of anxiety. Further research is needed for individual and organisational factors which protect officers from anxiety and for policies to reduce work stress.

\section{CORRECTION FOR REPORTING BIAS: THE IMPORTANCE OF STRATUM SPECIFIC ESTIMATES}

${ }^{1}$ Nicola Cherny, 'Jeremy Beach, ${ }^{1,2}$ Igor Burstyn, ${ }^{1}$ Ambikaipakan Sentilselvan. ${ }^{1}$ University of Alberta, Edmonton, Alberta, Canada; ${ }^{2}$ Drexel University, Philadelphia, USA

\subsection{6/oemed-2014-102362.9}

Objectives To examine whether reporting bias was responsible for increasing rates of self reported heart disease (SRHD) with exposure to phenoxy herbicides in a cohort of elderly grain farmers in Alberta.

Method We estimated exposure to chemical ingredients from named pesticides reported at interview after a lifetime of farming. Phenoxy exposure was grouped by tertiles as none, 1-22 yrs, 23-34 yrs and 35 yrs or greater. Six years after interview consent was sought from surviving farmers to link questionnaire data to provincial physician billing records. Sensitivity (Se) and specificity $(\mathrm{Sp})$ were estimated for SRHD, overall and within exposure stratum, and adjusted odds ratios (ORs) calculated.

Results Among the cohort of 2426, 373 had SRHD: ORs, adjusted for confounding, were estimated for phenoxy tertile (compared with no exposure) as 1.32 (95\% CI 0.79-2.23), 1.67 (0.99-2.85), 2.03 (1.20-3.45). For the 1371 farmers consenting to record linkage, ORs before adjustment or correction were $1.25,1.43,2.69$. Comparing SRHD to physician billing diagnoses of heart disease gave an overall Se 0.49 and Sp 0.98. ORs were increased to $1.37,1.65,4.01$ when corrected by these $\mathrm{Se}$ and $\mathrm{Sp}$ estimates. Differences were seen in stratum specific Se but not Sp, with significantly lower Se (0.29: 95\% CI $0.15-0.48)$ in the group with no exposure. Applying stratum specific correction reduced ORs in all three categories $(0.55$, $0.82,1.29$ ).

Conclusions The correction approach used, which demonstrated the importance of stratum specific estimates, assumed no error in the validation data. Sensitivity analyses to explore this limitation will also be presented.

\section{UPPER AIRWAYS CANCER, MYELOID LEUKAEMIA AND OTHER CANCERS IN CHEMICAL WORKERS EXPOSED TO FORMALDEHYDE}

David Coggon, Georgia Ntani, Clare Harris, Keith Palmer. University of Southampton, Southampton, UK

10.1136/oemed-2014-102362.10

Objectives The International Agency for Research on Cancer controversially has classified formaldehyde as a cause of 
nasopharyngeal carcinoma and myeloid leukaemia. To provide further information on the risks of cancer from formaldehyde, we extended follow-up of 14008 male chemical workers at six factories in England and Wales.

Method The cohort was identified from employment records, and exposures to formaldehyde were classified on the basis of job title. Subjects were traced through health service records, and their mortality was compared with national death rates by the person-years method. Associations of exposure with incident upper airways cancer and leukaemia were further explored in nested case-control analyses.

Results More than 2000 additional deaths had occurred since last follow-up of the cohort. Excess deaths were observed from cancers of the oesophagus (100 v 93.2 expected), stomach (182 v 141.1), rectum ( $107 \mathrm{v} 86.8)$, liver (35 v 26.9) and lung $(813 \mathrm{v}$ 645.6), but none of these tumours exhibited a clear exposureresponse relationship. In nested case-control analyses of 115 men with upper airways cancer (including one nasopharyngeal cancer), 92 with leukaemia, and 45 with myeloid leukaemia, there were no elevations of risk in the highest exposure category $(>2$ ppm for $\geq 1$ year). When the two highest exposure categories were combined, the odds ratio for myeloid leukaemia was 1.26 (95\% CI 0.39-4.08).

Conclusions Our results provide no support for a hazard of myeloid leukaemia, nasopharyngeal carcinoma or other upper airways cancer from formaldehyde, and indicate that any excess risk of these diseases, even from relatively high exposures, is at most small.

\section{JOINT ASSOCIATION OF SMOKING AND SILICA DUST WITH LUNG CANCER RISK: A POPULATION-BASED CASE-REFERENT STUDY IN HONG KONG MEN}

Ignatius Tak-sun Yu, Lap Ah (Shelly) Tse. JC School of Public Health and Primary Care, The Chinese University of Hong Kong, Hong Kong, China

\subsection{6/oemed-2014-102362.11}

Objectives To examine the joint association of smoking and silica dust exposure with lung cancer risk.

Method This is a population-based case-referent study among Hong Kong Chinese males. We consecutively recruited 1208 newly diagnosed lung cancer cases and 1069 age-matched community referents during the period 2004-2006. We obtained each participant's lifelong smoking data, occupational history, and other important information including family cancer history. Unconditional multiple logistic regression analysis was performed to estimate the odds ratio (OR) and the 95\% confidence interval $(95 \% \mathrm{CI})$. We examined the joint association and tested potential interaction under multiplicative or additive risk model.

Results A total of 132 (10.9\%) lung cancers and 536 (50.1\%) community referents were never smokers. The prevalence of silica dust exposure among ever smokers was higher than the never smokers for both the lung cancers $(13.5 \%$ vs. $7.6 \%)$ and community referents $(6.4 \%$ vs. $3.7 \%)$. A 2.4 -fold $(95 \%$ CI: 1.05-5.52) risk of lung cancer for silica dust exposure was observed in never smokers, whilst it increased sharply to 12 -fold (95\% CI: 7.59-18.95) if the dust exposed workers were also smokers. A possible additive interaction was indicated between these two exposures but power is limited (synergy index $=1.61$, 95\% CI: $0.95-2.73$ ), particularly for the adenocarcinoma (synergy index $=1.25,95 \% \mathrm{CI}: 0.52-3.01)$.
Conclusions Our findings supports the conclusion of IARC that workers exposed to silica dust increase lung cancer risk but adds new evidence on a positive additive interaction between silica and smoking. [Research Grants Council (Project CUHK4460/ 03M), Hong Kong].

\section{WORK INTENSITY, INJURY, STRESS AND PAIN AMONG COMMERCIAL JANITORS}

Noah Seixas, Bert Stover, Nancy Simcox, Carlos Dominguez. University of Washington, Seattle, WA, USA

\subsection{6/oemed-2014-102362.12}

Objectives Commercial janitors are a relatively unseen and understudied segment of the workforce. Janitors report increasing work pressures over the past few years. We conducted a cross-sectional survey among janitors to evaluate the association of changes in work intensity and other aspects of work organisation on injury, musculoskeletal pain, disability, and stress.

Method We conducted a cross-sectional survey among commercial janitors, including both union and non-union workers, and a comparison group of union security guards using peer interviewers and electronic data collection. Work intensity was measured using a 10-point scale and outcomes including injury, musculoskeletal pain, disability, and stress were assessed for the current year, and two previous years. The association between work intensity and each outcome was evaluated, controlling for group and demographics.

Results Surveys were collected among 276 union and 78 nonunion janitors, and 76 security guards, $76 \%$ of whom were immigrants. An increase in work intensity among union janitors, and strong trend of increasing injury, pain, upper extremity disability and stress associated with work intensity was observed. Union janitors report an increase in injury over the past 3 years from 6.3 to $13.5 \%$. Multi-variable models further explore the impact of demographics and work characteristics on the increase in risk.

Conclusions Anecdotal reports of increased workload among janitors are substantiated by the reported increase in work pressure over the past three years and its association with stress, pain and injury among janitors.

\section{TIME OF EXPOSURE AND RISK OF ASBESTOS RELATED LUNG CANCER}

Bengt Järvholm, Evelina Åström. Umeå University, Umeå, Sweden

10.1136/oemed-2014-102362.13

Objectives It is well known that the risk of lung cancer decrease in ex-smokers some years after exposure have stopped and some studies indicate that the risk of asbestos related lung cancer decrease some years after the exposure had stopped. We have studied how the time after stop of exposure has influenced the risk of asbestos related lung cancer.

Method The incidence of lung cancer was studied in Swedish construction workers who had participated in health controls. The exposure to asbestos was estimated through the occupational titles. Occupational groups with highest risk of malignant mesothelioma were considered to be highly exposed to asbestos and the group with the lowest risk of malignant mesothelioma was considered to have the lowest exposure to asbestos. 